\title{
Thermo-responsive graphene dispersions by liquid phase exfoliation of graphite aided by an alkylated Percec monodendron
}

\author{
Keyang Yin ${ }^{1,2}$, Qian Liu ${ }^{1}$, Liping Wang ${ }^{1 *}$, Shengju Zhou ${ }^{2,3}$, Baoyong Liu ${ }^{2,3}$ and Hongguang Li $^{2 *}$
}

\begin{abstract}
Large-scale production of graphene and subsequent sample engineering is the key for fully-realizing the potential applications proposed to this intriguing two-dimensional nanomaterial. Herein, smart graphene dispersions with low defects and thermo-responsive properties can be obtained by liquid phase exfoliation of graphite using an alkylated Percec monodendron (3,4,5-trioctadecyloxybenzaldehyde, 1) as the stabilizing reagent. By simply changing the temperature, the dispersed graphene and 1 can be detached, leading to the recovery of both components. Besides noncovalent wrapping, the stabilizing reagent 1 can be also covalently attached to graphene through $[3+2] \mathrm{cy}-$ cloaddition. The covalently functionalized graphene sheets show improved dispersibility in organic solvents compared to the pristine graphene, which opens the door for their applications in various polymer matrixes. The strategy demonstrated here provides a new methodology to get smart graphene dispersions with multiple functions.
\end{abstract}

Keywords: graphene, thermo-responsive, liquid phase exfoliation, alkylated, cycloaddition

\section{INTRODUCTION}

In the past decade, there has been an enthusiasm on the investigation of two-dimensional nanomaterials with atomiclevel thickness. As the pioneer of this family, graphene has received considerable attention due to its unique electrical, mechanical and photothermal properties, and a variety of applications have been proposed in molecular devices, material and life sciences $[1,2]$. To fully realize these applications, scalable graphene production and subsequent sample engineering play a key role. Although micro-mechani- cal exfoliation (MME) of graphite and chemical vapor deposition (CVD) of carbon sources can produce large-area graphene with high quality, both of them suffer from the low production yield. Oxidation of graphite in mixed acids followed by chemical reduction facilitates large-scale production. However, the defects created by the oxidation cannot be fully removed by subsequent reduction, which is a shortcoming especially if the optoelectronic applications of graphene are concerned.

In recent years, much attention has been paid to liquid phase exfoliation (LPE) of graphite in organic solvents [3-11]. The as-obtained graphene has low defects and the dispersions are expected to be directly applied in electronic devices. However, some challenges still remain. First, the most effective organic solvents for graphite exfoliation normally have high boiling points and are difficult to be completely removed [6]. Second, even the most effective solvent (such as $\mathrm{N}$-methyl-2-pyrrolidone, NMP) is used, the concentration of graphene $\left(c_{\mathrm{g}}\right)$ in the final dispersion is not high enough to fulfill various applications. Although this problem can be partially solved by long-term $[7,8]$ or multiple [9] sonication, a gradual increase of the defect sites is noticed. Third, even though the graphene dispersions can be directly applied in solution-processed devices through spin-coating, it becomes difficult when solid samples are needed such as in the fabrication of electrode. Although graphene films can be obtained by filtration [5], one has to deal with a large volume of dispersion due to the low $c_{g}$, which could be a technological obstacle in practical applications. This problem also holds true for the

\footnotetext{
${ }^{1}$ College of Materials Science and Engineering, Liaocheng University, Liaocheng 252059, China

${ }^{2}$ State Key Laboratory of Solid Lubrication \& Laboratory of Clean Energy Chemistry and Materials, Lanzhou Institute of Chemical Physics, Chinese Academy of Sciences, Lanzhou 730000, China

${ }^{3}$ University of Chinese Academy of Sciences, Beijing 100049, China

*Corresponding authors (emails: wangliping5@163.com (Wang L); hgli@licp.cas.cn (Li H))
} 
recently-developed, shear-assisted production of graphene [12].

An alternative way to improve $c_{\mathrm{g}}$ is to use stabilizing reagents (SRs) [13-17]. This strategy can also greatly expand the range of solvents used for the exfoliation of graphite, even in aqueous solutions [18]. Typical SRs include alkylated compounds with different functional groups [13-15], polymers [16] and porphyrin derivatives [17]. For the alkylated compounds attempted up to now, the molecular structures are limited, i.e., only molecule bearing one aliphatic chain has been tried. Moreover, the SRs can be hardly removed, and the alkyl chains on the graphene surface may serve as an insulating layer which significantly influences the electrical property of graphene.

Herein we report the preparation of graphene dispersions through LPE method aided by a typical alkylated Percec monodendron (APM) which bears three long alkyl chains (3,4,5-trioctadecyloxybenzaldehyde, 1, Fig. 1a). Moreover, temperature-induced recovery of the dispersed graphene back to solids which can be easily separated by filtration has been demonstrated for the first time. Finally, the alkyl chains have also been covalently anchored onto the outer layer of the recovered graphene via [3+2] cycloaddition, which significantly improves the dispersibility of the functionalized graphene in various organic solvents.

\section{EXPERIMENTAL SECTION}

\section{Materitals}

3,4,5-trihydroxybenzaldehyde monohydrate (purity: 98\%) was purchased from Sigma-Aldrich. Sarcosine (purity: 99\%) was purchased from Shanghai Aladdin Biochemical Technology Co., Ltd. Graphite flakes (325 mesh) was obtained from Shanghai Chemical Company. Chlorobenzene (analytical grade) was from Tianjin Chemicals. Pristine graphene used for comparison was obtained from Nanjing XFNANO Materials Tech Co., Ltd. 1-Bromooctadecane was from Shanghai Three-friends Chemical Regent Co., Ltd (China). Other chemicals and solvents were from local suppliers with the quality of analytical grade. All the chemicals were used without further purification unless otherwise stated. High-purity water was obtained from a water purification system (Ulupure Instrument Co., Ltd). Synthesis and characterization of 3,4,5-trioctadecyloxy- $\mathbf{a}$

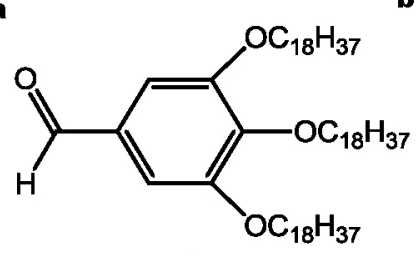

1 b

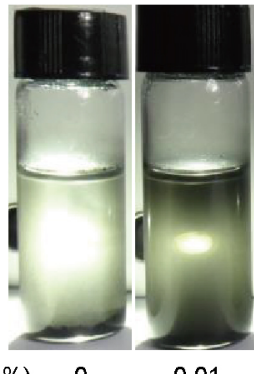

$c_{1}($ wt. \%) 0

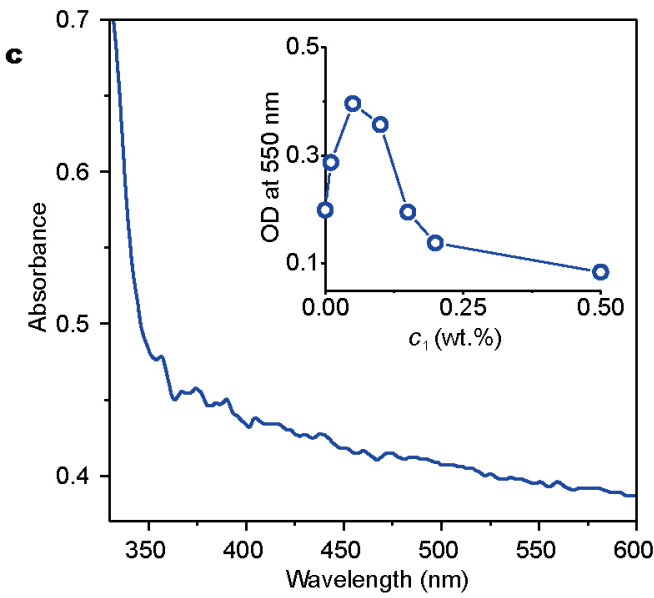

d

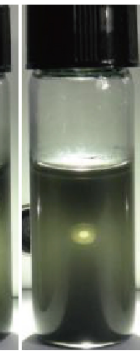

0.05

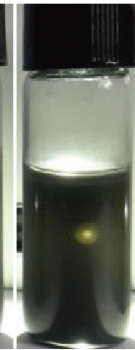

0.1

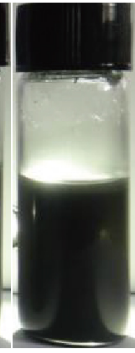

0.15

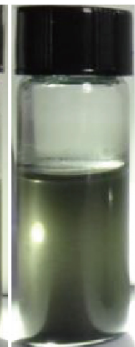

0.2

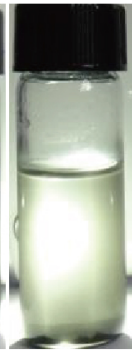

0.5

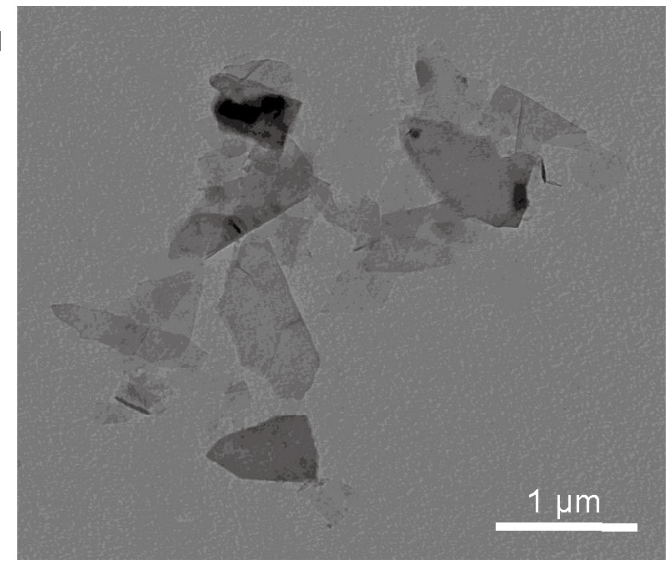

Figure 1 (a) Molecular structure of the alkylated Percec monodendron 1 ; (b) photos of the dispersions with $5 \mathrm{mg} \mathrm{mL}^{-1}$ graphite and increasing $c_{1}$ taken after sonicated at room temperature for $2 \mathrm{~h}$ and storage at $20^{\circ} \mathrm{C}$ for two weeks; (c) UV-vis absorption of a typical dispersion with $c_{1}=0.05$ wt. $\%$ (diluted to one third of the original $c_{\mathrm{g}}$ ). Inset is the variation of the optical density (OD) at $550 \mathrm{~nm}$ of the diluted dispersion as a function of $c_{1 . ;}$ (d) a typical TEM image of G-d. 
benzaldehyde (1) can be found in the Supplementary information.

\section{Liquid phase exfoliation of graphite}

To a series of glass bottles containing $5 \mathrm{mg} \mathrm{mL}^{-1}$ graphite in chlorobenzene (15 $\mathrm{mg}$ in $3 \mathrm{~mL}$ ), varying amount of 1 was added. The mixtures were then sonicated $(42-45 \mathrm{kHz}$, $80 \mathrm{~W}$ ) for $2 \mathrm{~h}$ at room temperature, during which the water in the ultrasonicator was updated every half an hour to avoid significant temperature rise caused by sonication. The as-prepared dispersions were allowed to stand for two weeks in an incubator at $20^{\circ} \mathrm{C}$ for the sedimentation of large particles before further characterizations.

For the experiments aiming to see the effects of sonication time and temperature, the concentration of $\mathbf{1}$ was fixed at 0.15 wt.\%. Four temperatures were selected, i.e., 25, 35, $45,55^{\circ} \mathrm{C}$, and the time of sonication was fixed at 2,6 , and $12 \mathrm{~h}$, respectively. During sonication, the temperature in the ultrasonicator was checked every $5 \mathrm{~min}$ and wherever necessary water with lower temperatures was added to keep the desired temperature. The as-prepared dispersions were allowed to stand in an incubator at $20^{\circ} \mathrm{C}$ for the sedimentation of large particles and the observation time was prolonged to three weeks for a better differentiation between different experimental conditions.

Dispersions with larger amount $(\sim 400 \mathrm{~mL})$ for subsequent chemical functionalizations were prepared at $25^{\circ} \mathrm{C}$ for $12 \mathrm{~h}$ sonication with $5 \mathrm{mg} \mathrm{mL}^{-1}$ graphite and $0.15 \mathrm{wt} . \%$ 1.

\section{Sample preparation method for $\mathbf{G}-\mathbf{d}_{s}, \mathbf{G}-\mathbf{8 0}$ and G-0}

To obtain $\mathrm{G}-\mathrm{d}_{\mathrm{s}}$, a part of $\mathrm{G}-\mathrm{d}_{1}$ was first subjected to rotary evaporation followed by vacuum drying at $40^{\circ} \mathrm{C}$ for 2-3 d. For G-80, the phase separated sample was first filtered and then washed thoroughly by chlorobenzene which was pre-warmed to $\sim 50^{\circ} \mathrm{C}$. The solid sample was further dried under high vacuum at $40^{\circ} \mathrm{C}$ for $2-3 \mathrm{~d}$. G-0 was obtained following similar procedures with G-80, except that the chlorobenzene used for washing was pre-cooled in a refrigerator to $\sim 4^{\circ} \mathrm{C}$.

\section{Covalent functionalization of the dispersed graphene}

To a $500 \mathrm{~mL}$ round-bottom flask containing $240 \mathrm{~mL}$ graphene dispersions, $0.3905 \mathrm{~g}(4.384 \mathrm{mmol})$ sarcosine was added. The mixture was then stirred at $140^{\circ} \mathrm{C}$ for $4 \mathrm{~d}$ under Ar. After cooling to room temperature, the as-prepared functionalized graphene which deposited at the bottom was filtered out using polyvinyl fluoride (PVDF) membrane with a pore size of $0.45 \mu \mathrm{m}$ followed by thorough washing with chlorobenzene for at least 3 times to remove the unreacted 1. The solid was then vacuum dried for further characterizations.

\section{Instruments and methods}

Nuclear magnetic resonance spectra were recorded on a Bruker Avance 400 spectrometer (Bruker, Germany) operated at a deuterium frequency of $400 \mathrm{MHz}$ (for ${ }^{1} \mathrm{H}$ NMR). Electrospray ionization mass spectrometry (ESI-MS) was performed on a Q-TOF6510 spectrograph (Agilent, USA). UV-vis measurements were carried out on a computer-manipulated spectrometer (UV-vis 4100, Hitachi, Japan). Fourier transform infrared (FTIR) spectra were recorded on a VERTEX-70/70v spectrometer (Bruker Optics, Germany) using KBr pellets. Transmission electron microscopy (TEM) observations were conducted on a JEOL JEM-100 CXII (Japan) instrument operated at $100 \mathrm{kV}$. High-resolution TEM (HR-TEM) images were recorded on a HRTEM JEOL 2100 instrument operated at $200 \mathrm{kV}$. For sample preparation, a drop of diluted dispersion was placed on a holey-carbon coated copper grid (300 meshes) and dried by an infrared lamp. Raman spectra were obtained using an NXR FT-Raman module (Nexus 670 , Nicolet Co.) equipped with a Gedecetor. The samples were excited by a laster source with a wavelength of $633 \mathrm{~nm}$. Differential scanning calorimetry (DSC) measurements were obtained with a DSC-Q2000 differential scanning calorimeter thermal analysis system (TA instrument, USA). Samples were analyzed in aluminium crucibles under a flow of nitrogen and heated at $10^{\circ} \mathrm{C} \mathrm{min}^{-1}$. An empty aluminium crucible was used as a reference. Thermogravimetric analysis (TGA) were carried out with DSC 822e (Piscataway, New Jersey) under nitrogen with the scanning speed of $10^{\circ} \mathrm{C} \mathrm{min}^{-1}$.

\section{RESULTS AND DISCUSSION}

\section{Exfoliation of graphite at room temperature}

APMs [19] have been frequently used as the building blocks in supramolecular structures such as thermotropic liquid crystals [20]. They are also known to have strong affinities with the surface of highly oriented pyrolytic graphite (HOPG) [21]. In current work, chlorobenzene was selected as the solvent due to its popularity in the fabrication of optoelectronic devices such as organic solar cells. First, the effect of $\mathbf{1}$ on the exfoliation of graphite was investigated on the samples containing $5 \mathrm{mg} \mathrm{mL}^{-1}$ graphite $(3 \mathrm{~mL})$ and increasing amount of $\mathbf{1}\left(c_{1}\right)$. After sonicated for $2 \mathrm{~h}$ (42-45 kHz, $80 \mathrm{~W})$ at room temperature followed by storage at $20^{\circ} \mathrm{C}$ for two weeks, $c_{\mathrm{g}}$ in the supernatant was evaluated. As shown in Fig. 1b, the exfoliation of graphite 
without 1 is poor. With increasing $c_{1}$ (up to $0.15 \mathrm{wt} . \%$ ), the color of the supernatant becomes heavier, indicating an increase of $c_{\mathrm{g}}$. Thus it can be concluded that the presence of $\mathbf{1}$ can significantly facilitate the exfoliation of graphite. Further increasing $c_{1}$ to $0.2 \mathrm{wt}$.\% also produces a homogeneous dispersion immediately after sonication. However, a lower $c_{\mathrm{g}}$ was noticed after storage, indicating that the stability of the dispersion decreased. This trend continues when $c_{1}$ was increased to $0.5 \mathrm{wt} . \%$ where the supernatant is almost colorless after stored for two weeks. Similar trend has been noticed previously in aqueous dispersions of carbon nanotubes (CNTs) stabilized by a branched amphiphilic copolymer [22], where the reason was ascribed to the depletion effect caused by the micelle formation at high polymer concentrations. Here, this explanation does not seem to apply as $\mathbf{1}$ only forms a clear solution in chlorobenzene. We suppose that the increase of $c_{1}$ induces two effects. On one hand, the number of $\mathbf{1}$ available for each graphite particle increases, leads to an increased $c_{\mathrm{g}}$. On the other hand, pieces of graphite with larger sizes and/or number of layers can be obtained, leading to a decreased stability upon storage. In order to further verify the latter assumption, the stability of the dispersions upon dilution (to one third of the original $c_{\mathrm{g}}$ ) has been investigated. Although the sample with $c_{1}=0.15 \mathrm{wt}$. $\%$ has a larger $c_{\mathrm{g}}$ after storage (as evidenced by their heavier color shown in Fig. 1b), it shows a greater extent of precipitation upon dilution (Fig. 1c and Fig. S1, Supplementary information). For the sample with $c_{1}=0.1$ wt.\%, precipitation was also noticed but with a less extent. In contrast, samples with $c_{1}<0.1 \mathrm{wt}$.\% show good stability upon dilution, which could be ascribed to the presence of graphene with smaller sizes and/or number of layers.

It is known that in LPE method, prolonged sonication time can lead to the exfoliation of a larger extent of graphite. Meanwhile, the number of the graphene layers and the sizes of graphene could be reduced, leading to the improvement of both $c_{\mathrm{g}}$ and the stability of the dispersion. To further improve the quality of our graphene dispersions while at the same time, avoid significant destruction of the graphene lattice, the sonication time was prolonged from 2 to $12 \mathrm{~h}$ for a typical sample with $0.15 \mathrm{wt} . \% 1$ and $5 \mathrm{mg} \mathrm{mL}^{-1}$ graphite. After being stored at $20^{\circ} \mathrm{C}$ for three weeks, the graphene dispersion (this sample is denoted as G- $\mathrm{d}_{1}$ hereafter) still exhibits much heavier color compared to that prepared at $2 \mathrm{~h}$ sonication and it is also stable against dilution. UV-vis measurement revealed a more than eight-fold increase of the optical density (OD) at $550 \mathrm{~nm}$ (Fig. S2). Based on TGA of graphite, 1 and the dried sample of G- $\mathrm{d}_{1}$ (denoted as $\left.\mathrm{G}-\mathrm{d}_{\mathrm{s}}\right), c_{\mathrm{g}}$ was determined to be $\sim 0.18 \mathrm{mg} \mathrm{mL}^{-1}$, which is among the highest values for the dispersions prepared by LPE method under similar or even harsher experimental conditions (Table S1). It should also be emphasized that the amount of $\mathbf{1}$ used is much smaller compared to other SRs to get similar $c_{\mathrm{g}}$. For example, to get dispersions with $c_{\mathrm{g}}$ of 0.1 or $0.128 \mathrm{mg} \mathrm{mL}^{-1}$, up to $20 \mathrm{wt} . \%$ 1-phenyloctane or arachidic acid is needed [13], which is more than 133 times larger than $\mathbf{1}$ applied in current system. The high efficiency of $\mathbf{1}$ to exfoliate graphite can be ascribed to its branched structure, which creates a stronger affinity with the graphene surface and a better steric repulsion between neighbouring layers compared to its linear analogues [22].

Unlike the MME or CVD method, LPE actually produces a mixture of exfoliated products. TEM observations on G- $\mathrm{d}_{1}$ show that it contains micrometer-sized graphene with varying shapes (Fig. 1d and Fig. S3). Besides the presence of a few multi-layered sheets, the majority of the graphene sheets contain only a few layers or just one layer as confirmed by the very low contrast. To get more information, the quality of the exfoliated graphene was further evaluated by Raman spectra. It is widely accepted that the $\mathrm{D}$ peak around $1350 \mathrm{~cm}^{-1}$ is caused by the defect sites of the graphene lattice. As shown in Fig. 2, graphite has a very small D peak with a D-to-G area ratio $\left(S_{\mathrm{D}} / S_{\mathrm{G}}\right)$ of 0.10 . For G- $\mathrm{d}_{s}$, a more pronounced D peak was noticed with a $S_{\mathrm{D}} / S_{\mathrm{G}}$ of 0.94 . The relatively low signal-to-noise ratio and the bulge centered around $2885 \mathrm{~cm}^{-1}$ should be caused by the presence of 1 which occupies over $85 \%$ by weight (TGA, see below). However, the influence of $\mathbf{1}$ on $S_{\mathrm{D}} / S_{\mathrm{G}}$, if there is any, should be small due to its much weaker Raman intensity

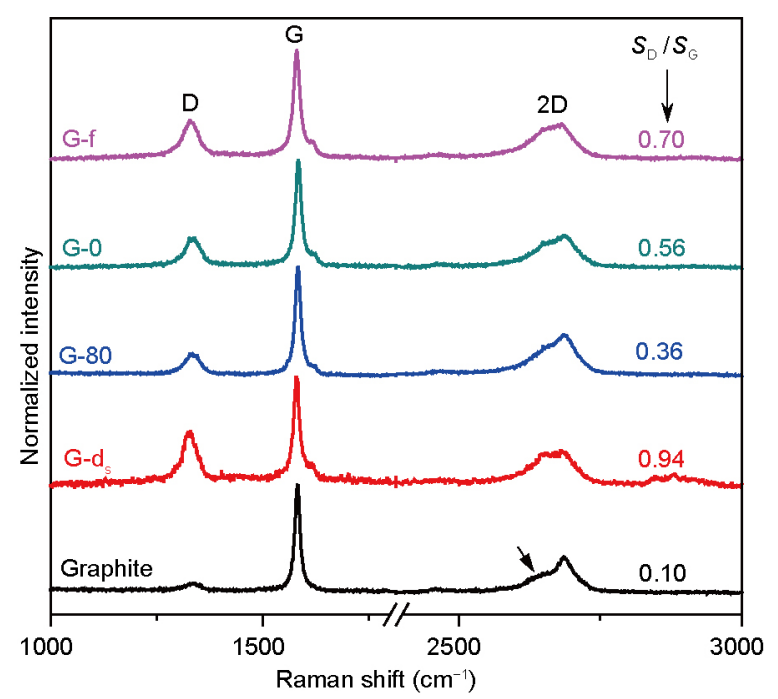

Figure 2 Raman spectra of graphite, dried sample from $\mathrm{G}-\mathrm{d}_{1}\left(\mathrm{G}-\mathrm{d}_{\mathrm{s}}\right)$, precipitates at $80^{\circ} \mathrm{C}(\mathrm{G}-80), 0^{\circ} \mathrm{C}(\mathrm{G}-0)$ and functionalized graphene (G-f). 
compared to graphene as evidenced by the absence of most of its characteristic peaks (Fig. S4) in G- $\mathrm{d}_{s}$. Thus, the much larger $S_{\mathrm{D}} / S_{\mathrm{G}}$ of G-d $\mathrm{d}_{\mathrm{s}}$ compared to that of graphite can be mainly ascribed to the effect of sonication. Compared to the samples obtained by the LPE method at the presence of linear SRs [13-15], this $S_{\mathrm{D}} / S_{\mathrm{G}}$ value is smaller, which indicates a reduced number of defect sites and again highlights the advantage of using $\mathbf{1}$ as the SR for the exfoliation of graphite. The $2 \mathrm{D}$ peak is known to contain important information about the number of graphene layers [23]. For graphite, a shoulder peak was observed (indicated by the arrow), which is typical for stacked graphene layers. After exfoliation, this peak almost disappears totally, indicating that the number of the graphene layers has been greatly reduced.

\section{Thermo-responsive properties}

Previously, we have reported that the dispersion state of surfactant-wrapped CNTs can be controlled by the variation of temperature $[24,25]$. Considering the structural similarity between graphene and CNTs, such rule might be also applied in graphene dispersions. To test this assumption, the thermo-responsiveness of G- $\mathrm{d}_{1}$ was investigated. Our strategy to handle the sample can be schematically summarized in Fig. 3. When $\mathrm{G}-\mathrm{d}_{1}$ was heated to $\sim 80^{\circ} \mathrm{C}$, i.e., around $T_{\mathrm{c}}$ of $\mathbf{1}$ (Fig. 4), the homogeneous dispersion became to precipitate. This unstability of G- $\mathrm{d}_{1}$ can be ascribed to the desorption of $\mathbf{1}$ from the graphene lattice, which is reminiscent of the phenomenon observed in surfactant-wrapped CNTs $[24,25]$. In fact, the influence of temperature on the adsorption-desorption of $\mathbf{1}$ on the graphene surface can be already seen during the exfoliation of graphite. For the dispersions prepared at varying temperatures, it was found that the $c_{\mathrm{g}}$ in the final dispersion decreases continuously with increasing temperature as confirmed by UV-vis measurements (Fig. S5).

The precipitated graphene (denoted as G-80) can be facilely separated by filtration and subsequent washing, which yields a bulky paper (Fig. S6) with only trace of remaining 1 as confirmed by the rather low weight loss between 330 and $500^{\circ} \mathrm{C}$ in TGA ( $<5 \%$, Fig. 5a, Table S2). Evidence of the removal of $\mathbf{1}$ can be also found from FTIR spectra where the intensities of the asymmetric and symmetric methylene stretching for the alkyl chains around 2920 and $2851 \mathrm{~cm}^{-1}$ decrease significantly compared to $\mathrm{G}-\mathrm{d}_{\mathrm{s}}$ (Fig. 5b). Unlike previous method to get the bulky paper by directly filtering a large volume of the graphene dispersion [5], current strategy allows us to pour the clear supernatant beforehand, which significantly reduces the volume of the sample to be handled. More importantly, from Raman spectra in Fig. 2, it can be seen that $S_{\mathrm{D}} / S_{\mathrm{G}}$ of G-80 (0.36) is much smaller compared to that of G-d $\mathrm{d}_{s}$ (0.94), which indicates that the phase separation simultaneously purifies the graphene. It is possible that the tiny

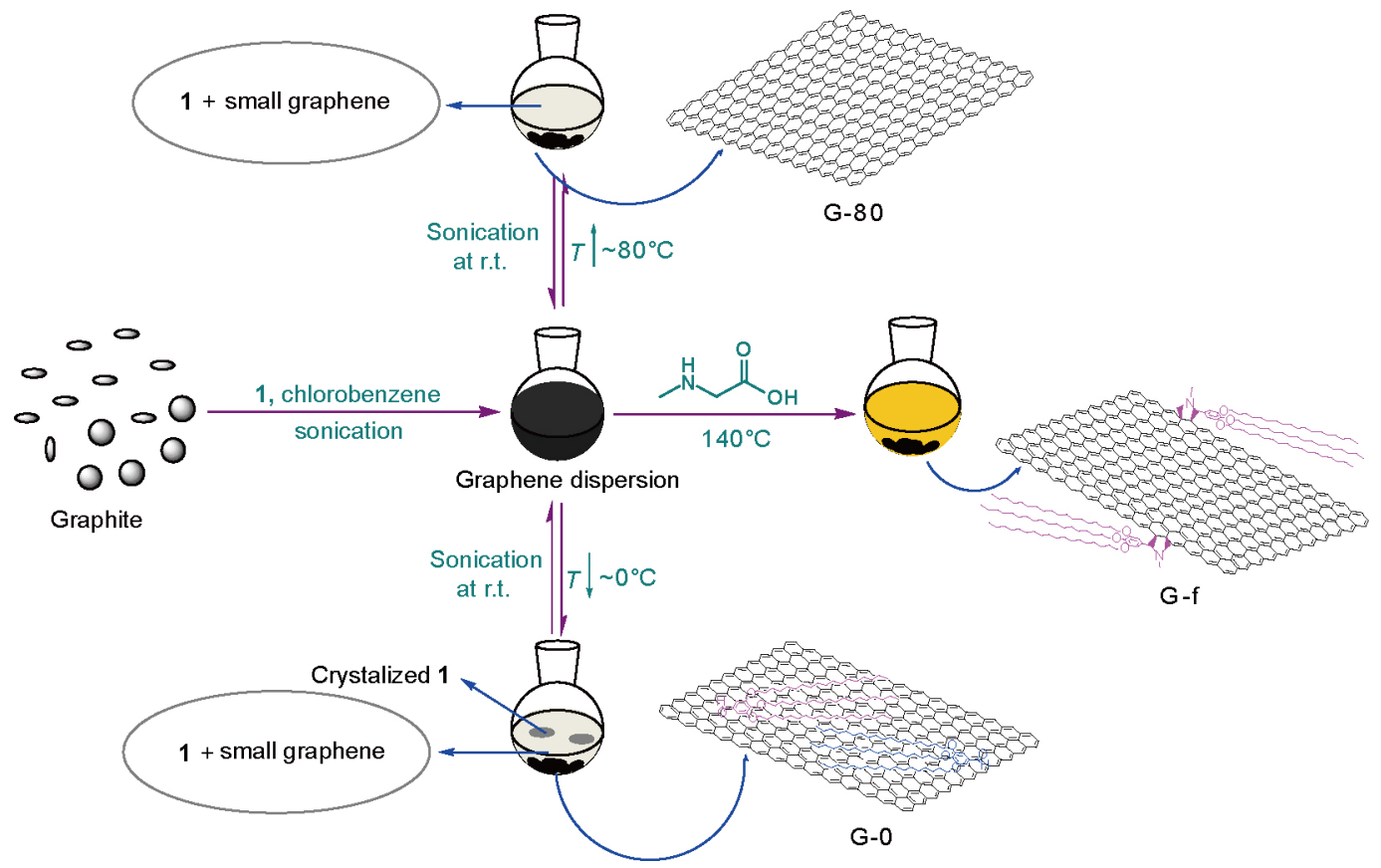

Figure 3 Illustration of the thermo-responsive characteristics of the graphene dispersion as well as the covalent functionalization of the dispersed graphene. 


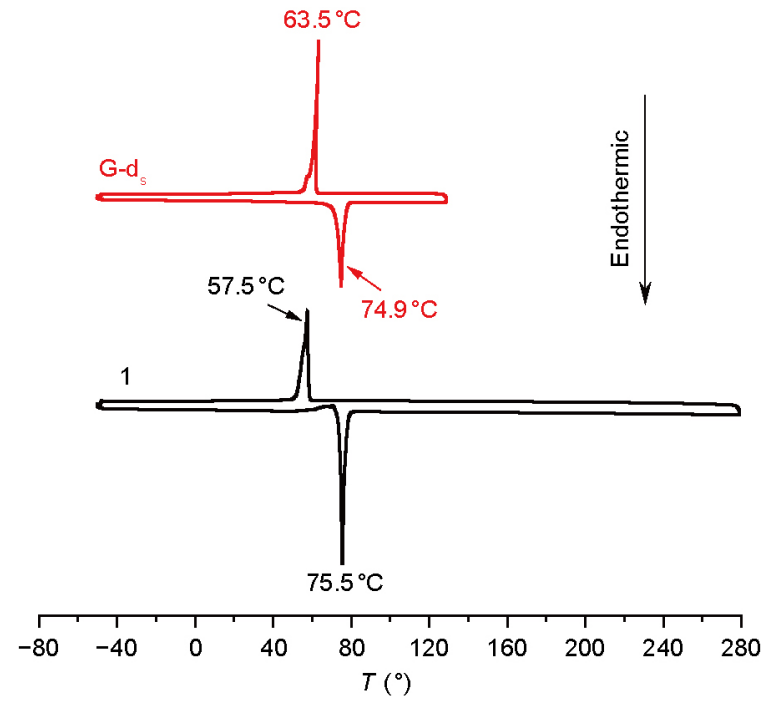

Figure 4 DSC curves from the second heating-cooling circles of $\mathbf{1}$ and $\mathrm{G}-\mathrm{d}_{\mathrm{s}}$. The temperatures at which the peaks appear have been indicated. fragments produced during the exfoliation of graphite, which have higher solubilities in chlorobenzene, remain in the supernatant and have been removed by pour-out or filtration. To verify this assumption, a part of the supernatant has been dried and subjected to TGA. While 1 decomposes completely before $500^{\circ} \mathrm{C}$, there is still $7.96 \%$ weight remaining for the dried supernatant (Fig. S7), which is assigned to the tiny fragments.

If $\mathrm{G}-\mathrm{d}_{1}$ was cooled to $\sim 0^{\circ} \mathrm{C}$, precipitation also occurs. Meanwhile, solids which are assigned to the crystalized 1 are found at the top of the sample. Thus, the precipitation is likely to be caused by the decreased solubility of $\mathbf{1}$ in chlorobenzene. In this case, the precipitates should be graphene with adsorbed $\mathbf{1}$ (denoted as G-0). Indeed, the amount of remaining $\mathbf{1}$ in G-0 after washing is larger compared to G-80, as confirmed by the more pronounced asymmetric and symmetric methylene stretching shown in FTIR (Fig. 5b) and the larger weight loss between 330 and
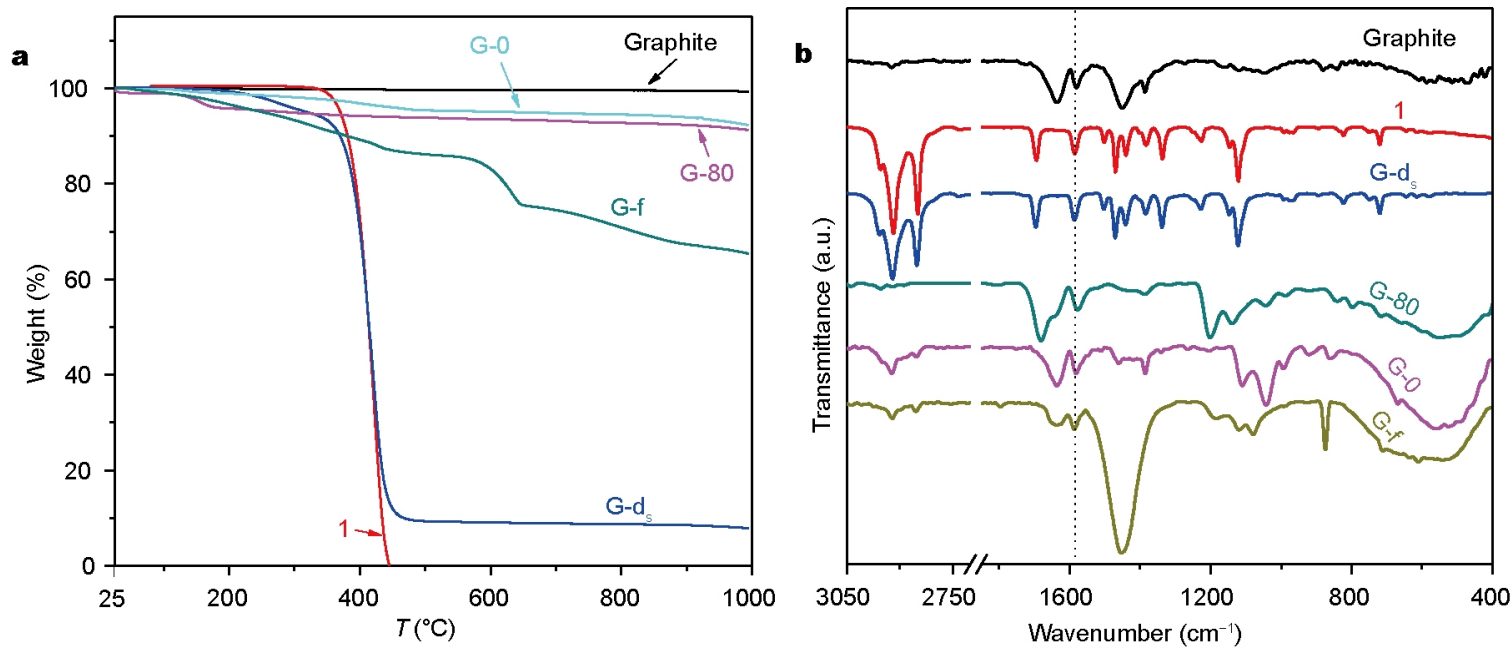

c
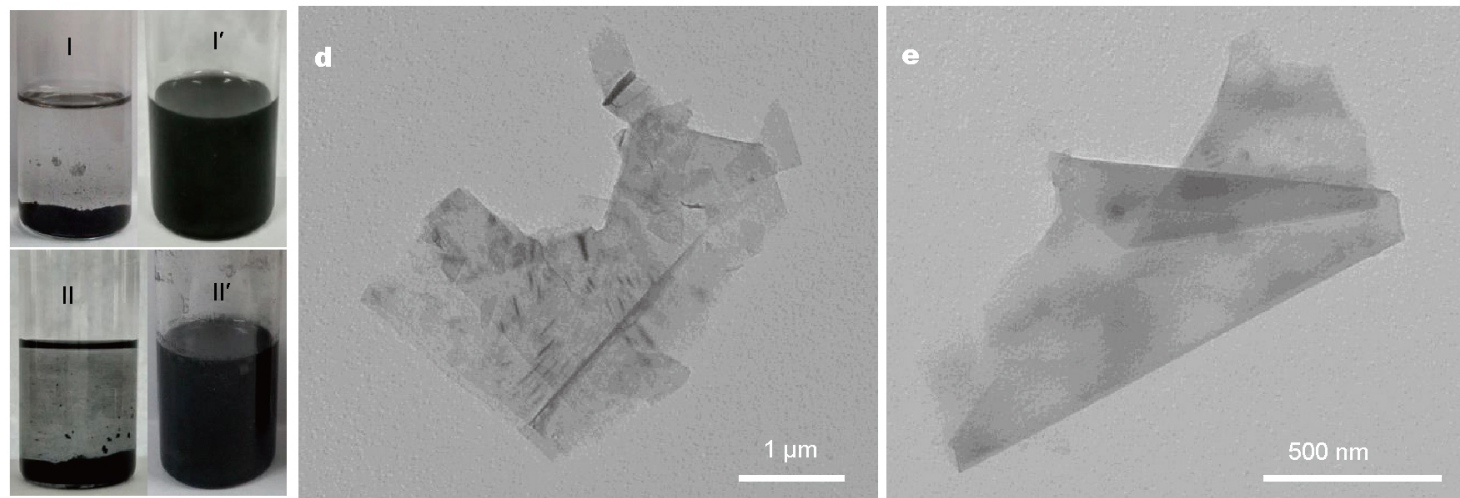

Figure 5 (a) TGA of selected solid samples as indicated; (b) FTIR of various solid samples as indicated. Normalized by the peak around $1581 \mathrm{~cm}^{-1}$ (the dotted line); (c) photos of $\mathrm{G}-\mathrm{d}_{1}$ treated at $80^{\circ} \mathrm{C}$ (I) and $0^{\circ} \mathrm{C}$ (II) followed by re-dispersion by sonication for $1 \mathrm{~h}\left(\mathrm{I}^{\prime}\right.$, II'); (d, e) TEM images of the sample $\mathrm{I}^{\prime}$ and $\mathrm{II}^{\prime}$. 
$500^{\circ} \mathrm{C}$ in TGA (Table S2). Raman spectrum gives a $S_{\mathrm{D}} / S_{\mathrm{G}}$ of 0.56 , which is also smaller compared to that of $\mathrm{G}-\mathrm{d}_{\mathrm{s}}$. TGA of the dried supernatant indicates that $7.72 \%$ weight is remaining at $500^{\circ} \mathrm{C}$ (Fig. S7), indicating that the graphene involved in G-0 has also been purified by the phase separation albeit with a lesser extent compared to G-80. To check the reversibility of the thermo-responsiveness of G- $\mathrm{d}_{1}$, the phase separated samples obtained at 80 and $0^{\circ} \mathrm{C}$ (before filtration) were re-sonicated for $\sim 1 \mathrm{~h}$. Homogeneous samples were obtained which can be stable for more than $4 \mathrm{~h}$ (Fig. 5c). TEM observations revealed the presence of re-stacked or plicated graphene (Fig. $5 \mathrm{~d}, \mathrm{e}$ ).

\section{Covalent functionalization}

Chemically functionalized graphene has wide applications in the preparation of hybrid materials. Aldehyde is wellknown to be reactive with curved or planar carbon lattices through [3+2] cycloaddition at the presence of glycine derivatives. This reaction was first carried out on fullerene $\mathrm{C}_{60}$ (i.e., the Prato reaction) $[26,27]$ and later extended to CNTs [28-30] and graphene [31,32]. In current system, the graphene is stabilized by an aldehyde derivative (i.e., $\mathbf{1}$ ) whose analogues have been used to functionalize fullerene $\mathrm{C}_{60}$ via Prato reaction [21]. Although at elevated temperatures, $\mathbf{1}$ tends to desorb from the graphene surface, it is possible that covalently functionalization can still occur on the outer layer of the recovered graphene. To test this assumption, the mixture was refluxed for $\sim 4 \mathrm{~d}$ after introduction of sarcosine (ten times that of $\mathbf{1}$ ). When cooled to room temperature, black precipitates formed together with a color change of the supernatant to yellow. The black precipitates were then separated by filtration and washed (denoted as G-f). In TGA, a multi-step weight loss was observed with a much higher total weight loss compared to G-80 and G-0 (Fig. 5a). Besides the more pronounced weight loss between 330 and $500^{\circ} \mathrm{C}$, considerable weight loss was also noticed above $500^{\circ} \mathrm{C}$ (Table S2). This indicates that the chemical functionalization destabilizes the skeleton of graphene through destroying the $\mathrm{C}=\mathrm{C}$ double bonds. Raman spectrum gives a $S_{\mathrm{D}} / S_{\mathrm{G}}$ of 0.70 (Fig. 2), which is much larger than that of G-80 and G-0. These results indicate that more defect sites have been created in the graphene lattice, which should be ascribed to the [3+2] cycloaddition.

The covalent attachment of alkyl chains onto the graphene lattice imparts G-f good dispersibility in organic solvents, especially in 1,4-dioxane. After sonicated for $2 \mathrm{~h}$, a homogeneous dispersion can be obtained which can be stored for at least $4 \mathrm{~h}$ with only limited precipitation (Fig. 6a). Even after $24 \mathrm{~h}$, the supernatant still remains black. Unlike the re-dispersion of G-80 and G-0, in this case there is no $\mathbf{1}$ acting as the SR. In contrast, for commercially available pristine graphene, obvious phase separation can be observed $1 \mathrm{~h}$ after sonication, while for graphite, phase separation occurs $10 \mathrm{~min}$ after sonication. The improved dispersibility of G-f is advantageous for engineering graphene with various hosts such as polymers, during which pre-dispersion in an organic solvent is required. As can be understood, the type of solvent has a big influence on the dispersibility of G-f (Fig. S8). In chlorobenzene and isopropanol, the dispersions can be stable for $\sim 1 \mathrm{~h}$. This

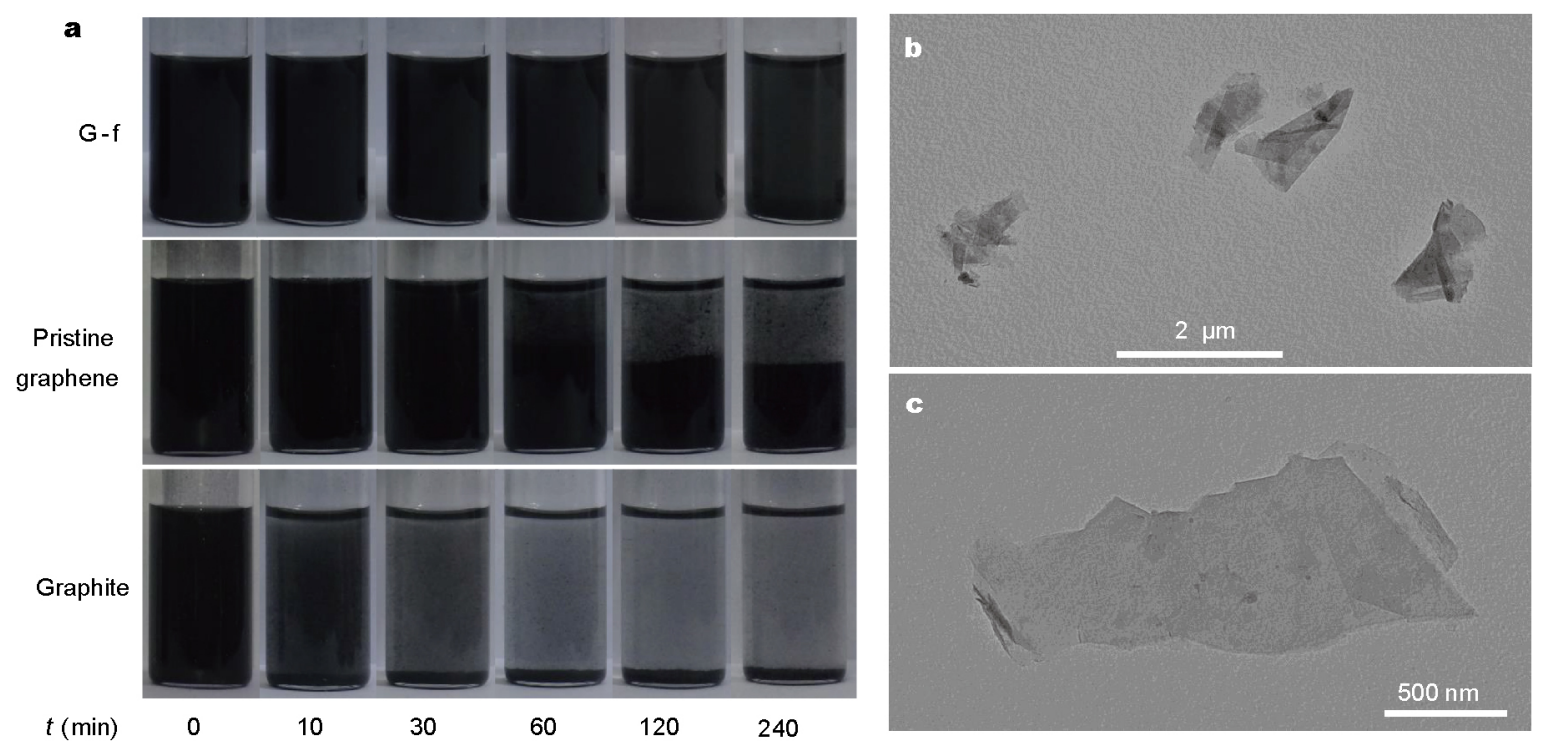

Figure 6 (a) Photos of dispersions in 1,4-dioxane (sonicated for 2 h) of G-f, pristine graphene and graphite taken at varying time; (b, c) TEM images of the dispersion of G-f in 1,4-dioxane: (b) overview of four plicated graphene; (c) a piece of outstretched graphene. 
time decreases to $\sim 10 \mathrm{~min}$ in cyclohexane and toluene. The worst stability was found in DMF where G-f can be hardly dispersed. TEM observations on the dispersion of G-f in 1,4-dioxane reveal the presence of large area graphene (Fig. $6 c)$, indicating that the general structure of graphene retains after $[3+2]$ cycloaddition.

\section{CONCLUSIONS}

In summary, we have demonstrated that by using an alkylated Percec monodendron as the SR, smart graphene dispersions with thermo-responsive characteristics can be obtained by LPE method. The graphene has few defects and can be effectively recovered by changing temperature. During this process, the purity of the graphene can be further improved and $\mathbf{1}$ can be recycled. Besides noncovalent wrapping, the alkyl chains can be also in situ introduced to the graphene lattice through covalent bonds, which improves the dispersibility of the functionalized graphene in various organic solvents. Considering that Percec monodendrons have diverse molecular structures and can be easily synthesized, the strategy demonstrated here can pave a new way to facilely produce and engineer graphene for a wide range of applications.

\section{Received 19 December 2016; accepted 16 February 2017; published online 17 March 2017}

1 Roy-Mayhew JD, Aksay IA. Graphene materials and their use in dye-sensitized solar cells. Chem Rev, 2014, 114: 6323-6348

2 Georgakilas V, Tiwari JN, Kemp KC, et al. Noncovalent functionalization of graphene and graphene oxide for energy materials, biosensing, catalytic, and biomedical applications. Chem Rev, 2016, 116: 5464-5519

3 Coleman JN. Liquid exfoliation of defect-free graphene. Acc Chem Res, 2013, 46: 14-22

4 Parviz D, Irin F, Shah SA, et al. Challenges in liquid-phase exfoliation, processing, and assembly of pristine graphene. Adv Mater, 2016, 28: 8796-8818

5 Hernandez Y, Nicolosi V, Lotya M, et al. High-yield production of graphene by liquid-phase exfoliation of graphite. Nat Nanotech, 2008, 3: 563-568

6 Hernandez Y, Lotya M, Rickard D, et al. Measurement of multicomponent solubility parameters for graphene facilitates solvent discovery. Langmuir, 2010, 26: 3208-3213

7 Khan U, O'Neill A, Lotya M, et al. High-concentration solvent exfoliation of graphene. Small, 2010, 6: 864-871

8 O'Neill A, Khan U, Nirmalraj PN, et al. Graphene dispersion and exfoliation in low boiling point solvents. J Phys Chem C, 2011, 115: 5422-5428

9 Khan U, Porwal H, O’Neill A, et al. Solvent-exfoliated graphene at extremely high concentration. Langmuir, 2011, 27: 9077-9082

10 Hamilton CE, Lomeda JR, Sun Z, et al. High-yield organic dispersions of unfunctionalized graphene. Nano Lett, 2009, 9: 3460-3462

11 Bourlinos AB, Georgakilas V, Zboril R, et al. Liquid-phase exfoliation of graphite towards solubilized graphenes. Small, 2009, 5: 1841-1845
12 Paton KR, Varrla E, Backes C, et al. Scalable production of large quantities of defect-free few-layer graphene by shear exfoliation in liquids. Nat Mater, 2014, 13: 624-630

13 Ciesielski A, Haar S, El Gemayel M, et al. Harnessing the liquid-phase exfoliation of graphene using aliphatic compounds: a supramolecular approach. Angew Chem Int Ed, 2014, 53: 10355-10361

14 Haar S, Ciesielski A, Clough J, et al. A supramolecular strategy to leverage the liquid-phase exfoliation of graphene in the presence of surfactants: unraveling the role of the length of fatty acids. Small, 2015, 11: 1691-1702

15 Haar S, Bruna M, Lian JX, et al. Liquid-phase exfoliation of graphite into single- and few-layer graphene with $\alpha$-functionalized alkanes. J Phys Chem Lett, 2016, 7: 2714-2721

16 Wajid AS, Das S, Irin F, et al. Polymer-stabilized graphene dispersions at high concentrations in organic solvents for composite production. Carbon, 2012, 50: 526-534

17 Geng J, Kong BS, Yang SB, et al. Preparation of graphene relying on porphyrin exfoliation of graphite. Chem Commun, 2010, 46: 5091-5093

18 Lotya M, Hernandez Y, King PJ, et al. Liquid phase production of graphene by exfoliation of graphite in surfactant/water solutions. J Am Chem Soc, 2009, 131: 3611-3620

19 Rosen BM, Wilson CJ, Wilson DA, et al. Dendron-mediated selfassembly, disassembly, and self-organization of complex systems. Chem Rev, 2009, 109: 6275-6540

20 Binnemans K. Ionic liquid crystals. Chem Rev, 2005, 105: 4148-4204

21 Nakanishi T, Miyashita N, Michinobu T, et al. Perfectly straight nanowires of fullerenes bearing long alkyl chains on graphite. J Am Chem Soc, 2006, 128: 6328-6329

22 Xin X, Xu G, Zhao T, et al. Dispersing carbon nanotubes in aqueous solutions by a starlike block copolymer. J Phys Chem C, 2008, 112: $16377-16384$

23 Casiraghi C. Raman spectroscopy of graphene. In: Yarwood J, Douthwaite R, Duckett S (Eds.). Spectroscopic Properties of Inorganic and Organometallic Compounds: Techniques, Materials and Applications. Cambridge: the Royal Society of Chemistry, 2012, 29-56

24 Xin X, Li H, Wieczorek SA, et al. Incorporation of carbon nanotubes into a lyotropic liquid crystal by phase separation in the presence of a hydrophilic polymer. Langmuir, 2010, 26: 3562-3568

25 Xin X, Li H, Kalwarczyk E, et al. Single-walled carbon nanotube/lyotropic liquid crystal hybrid materials fabricated by a phase separation method in the presence of polyelectrolyte. Langmuir, 2010, 26: $8821-8828$

26 Maggini M, Scorrano G, Prato M. Addition of azomethine ylides to $\mathrm{C}_{60}$ : synthesis, characterization, and functionalization of fullerene pyrrolidines. J Am Chem Soc, 1993, 115: 9798-9799

27 Prato M, Maggini M. Fulleropyrrolidines: a family of full-fledged fullerene derivatives. Acc Chem Res, 1998, 31: 519-526

28 Georgakilas V, Kordatos K, Prato M, et al. Organic functionalization of carbon nanotubes. J Am Chem Soc, 2002, 124: 760-761

29 Tagmatarchis N, Prato M. Carbon-based materials: from fullerene nanostructures to functionalized carbon nanotubes. Pure Appl Chem, 2005, 77: 1675-1684

30 Campidelli S, Sooambar C, Lozano Diz E, et al. Dendrimer-functionalized single-wall carbon nanotubes: synthesis, characterization, and photoinduced electron transfer. J Am Chem Soc, 2006, 128: $12544-12552$

31 Quintana M, Spyrou K, Grzelczak M, et al. Functionalization of graphene via 1,3-dipolar cycloaddition. ACS Nano, 2010, 4: 
3527-3533

32 Georgakilas V, Bourlinos AB, Zboril R, et al. Organic functionalisation of graphenes. Chem Commun, 2010, 46: 1766-1768

Acknowledgments This work was supported by the Hundred Talents Program of Chinese Academy of Sciences (Y20245YBR1) and the National Natural Science Foundation of China (21402215 and 61474124). Prof. Wang thanks the financial support from Shandong Province Higher Education Science and Technology Program (J16LA01).
Author contributions Yin $\mathrm{K}$ performed the main part of the experiment with the help of Liu Q and wrote the draft. Zhou S and Liu B contributed to FTIR and TGA measurements, respectively. Wang L and $\mathrm{Li} \mathrm{H}$ designed the project, discussed the results and revised the paper.

Conflict of interest The authors declare that they have no conflict of interest.

Supplementary information Supporting data are available in the online version of the paper.
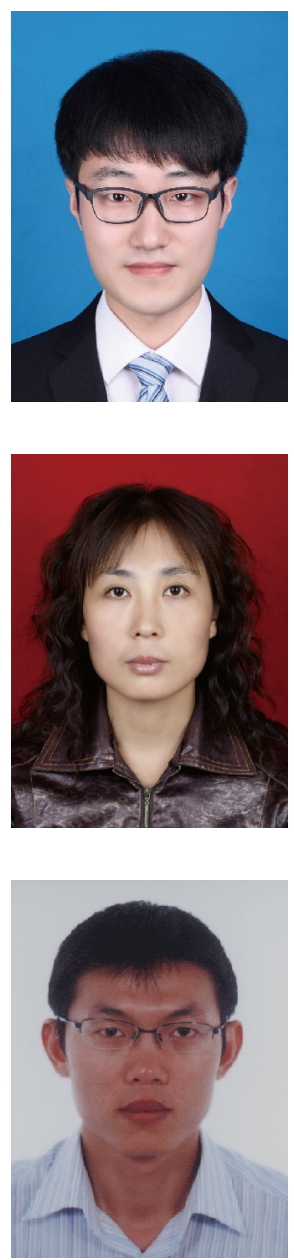

Keyang Yin was born in 1990. He obtained his BSc degree in chemistry education from Qilu Normal University in 2014, and he is currently a Master candidate at Liaocheng University. His research interests mainly focus on functional graphene, oil-soluble carbon quantum dots and their applications for energy-related devices.
Liping Wang was born in 1975. She obtained her BSc degree and PhD degree from Qufu Normal University in 1998 and Northwest Normal University in 2007 respectively. After two-year's postdoctoral research at Shandong University, she joined Liaocheng University. Her research interest mainly lies in "living" polymerization and honeycomb-patterned porous films.

Hongguang Li was born in 1979. He obtained his BSc degree and PhD degree from Shandong University in 2003 and 2008, respectively. After three-year's postdoctoral research, he joined the Laboratory of Clean Energy Chemistry and Materials, Lanzhou Institute of Chemical Physics, Chinese Academy of Sciences as a full-time professor. His current interest lies in the design of novel $\pi$-conjugated molecules and engineering carbon nanomaterials for applications in optoelectronic devices.

\section{烷基枝化分子辅助液相剥离石墨粉获得具有热响应的石墨烯分散液}

尹克样 ${ }^{1,2}$, 刘倩 ${ }^{1}$, 王利平 ${ }^{1^{*}}$, 周升菊 ${ }^{2,3}$, 刘宝勇 ${ }^{2,3}$, 李洪光 ${ }^{2 *}$

摘要 石墨烯的宏量制备及其后续处理工艺对全面实现这一有趣二维纳米材料的潜在应用起着至关重要的作用. 在此, 我们采用一种烷基 枝化的Percec型枝化分子(3,4,5-三十八烷基苯基醚-1-甲醛, 1)作为稳定剂, 利用液相剥离石墨粉的方法, 制备了具有低缺陷、热响应性能的 智能石墨烯分散液. 通过简单地改变温度, 便能实现处于分散状态的石墨烯与稳定剂 $\mathbf{1}$ 的分离和回收利用. 除了上述非共价缠绕, 稳定剂 $\mathbf{1}$ 也 能够通过[3+2]环加成反应共价修饰于石墨烯表面. 修饰后的石墨烯在多种有机溶剂中表现出比未修饰石墨烯更好的分散性, 从而为进一 步将其引入聚合物体系创造了条件. 本文所示的方法提供了一种制备智能石墨烯分散液以满足多种应用的新思路. 